\title{
LEÓN (1847-1900)
}

\section{Estefanía Fernández García}

\section{INTRODUCCIÓN ${ }^{1}$}

Cuenta la ciudad de León con una situación tan privilegiada, con el paso a Galicia, Asturias, Palencia, Burgos, etc., que compañías de gran prestigio actuaron en ella en sus idas y venidas a esos lugares. Ricardo de la Fuente Ballesteros (1988: 29), por ejemplo, cita entre las tres más famosas, a la de Vico, que estuvo en León en 1882 y la de Rafael Calvo en 1879.

Pero había muchas razones por las que León no era una plaza interesante para los grandes grupos teatrales: la escasa población que osciló entre los siete y los dieciocho mil habitantes, desde mediados a finales del siglo XIX, de los que sólo unos pocos podían permitirse el gasto de un abono de asistencia o del pago de las entradas; las reti-

${ }^{1}$ Para todo este artículo ver mi tesis doctoral: León y su actividad escénica en la segunda mitad del siglo XIX (Fernández García, 1997 y 2000). 
cencias para asistir al teatro dependiendo de la moralidad de ciertas compañías; la suspensión de las representaciones en Cuaresma -a pesar de no tener ya vigencia la prohibición general de las actuaciones en esta época, se cerró el teatro por orden gubernamental en 1877 y se denegó el permiso por los ediles en otras ocasiones-; la climatología, con los larguísimos inviernos; las vacaciones de la burguesía en verano; y la falta de interés por algunos espectáculos, limitaban la deseada asistencia.

La llegada del ferrocarril en 1863 favoreció la presencia de muchos grupos dramáticos de paso a otros lugares; pero ya en 1848 se documentan unas representaciones en León que se alternaban con las de $\mathrm{Pa}$ lencia.

La gestión municipal de los teatros se enmarca entre las posturas inmovilistas, que pretendían la intervención en todos los aspectos. Y así lo hizo el Ayuntamiento de León a lo largo de todo el siglo ${ }^{2}$. No dudaban en argumentar su interés en el control de todo el proceso para denegar algunas solicitudes de arriendo.

Las peticiones para un año teatral se denegaron o no hubo acuerdo en las negociaciones ${ }^{3}$. Era difícil mantener una temporada larga en una ciudad tan pequeña y con tan escaso poder adquisitivo.

La contratación de las compañías se hacía, siguiendo la tradición de siglos, en Madrid o en la vecina ciudad de Valladolid (Alonso Cortés, 1947). Solía pedirse información en los lugares en que hubiesen actuado con anterioridad.

El trato que recibían los diferentes grupos fue muy desigual: no sólo se escogía entre las diferentes solicitudes - se pidió un precio desmesurado a una compañía de acrobacia para favorecer a la compañía de Miguel Cepillo en una venida de Alfonso XII ${ }^{4}$ a la ciudad-, sino que algunos no pagaban el precio del alquiler o recibían sustanciosas subvenciones, como Antonio Vico. Una de las cláusulas más peregrinas que se recogen en las actas municipales fue la que sufrió la compañía de Luis Rivera, en 1855, que incluía la prohibición de dar funciones hasta que terminasen las rogativas contra el cólera morbo.

2 Hubo una corta excepción, cuando se le concedió a La Sociedad Económica de Amigos del País.

3 Se documenta un único contrato anual a la compañía de Antonio Solís, en los años cuarenta.

${ }^{4}$ La compañía había actuado en otra estancia del monarca en Valladolid (¿amores reales?). 
Las buenas compañías venían a León de paso y pocas veces al completo. No obstante recibían trato de favor. Las justificaciones a las preferencias municipales estaban cargadas de demagogia: era de difícil defensa mantener un local a cuenta del erario público y, además, concederlo gratis o subvencionarlo, o ambas cosas a la vez, para que unos pocos se divirtiesen. La de Antonio Vico hizo un atento reconocimiento de la subvención y exención del pago de los alquileres, en su estancia en la primavera de 1882. El Ayuntamiento pretextaba razones de peso:

El comercio, la industria de las poblaciones, se benefician con las estancias, aunque sean transitorias, de cincuenta familias (Archivo Histórico Municipal de León, Documento Teatro Principal, 03-09-1900).

A pesar de la privilegiada situación de estas agrupaciones, algunas se permitieron ciertas tropelías no muy acordes con la fama de la que venían precedidas: incumplimiento de contratos, suspensión de los espectáculos sin previo aviso o cuando ya estaba el público acomodado, actuaciones inadecuadas si la concurrencia era escasa y aplazamientos injustificados de las funciones o suspensión de las mismas para acudir a otros escenarios más interesantes ${ }^{5}$. Incluso se documentan impagos en los hospedajes; pero solía producirse con compañías de menor rango. El abandono de parte del elenco, que acababa a merced de la caridad pública, era frecuente. La compañía de Manuel Catalina, por ejemplo, dejó en la ciudad parte de su equipo en su estancia de 1884. Restos de la de Carolina Civili se vieron obligados a hacer una función con la que allegar fondos para abandonar la ciudad.

Entre las condiciones impuestas en los contratos figuraba la obligatoriedad de las compañías del envío de la lista de actores y los repertorios, y la sugerencia para la contratación de distintos actores o actrices. La exigencia de garantía económica para el cumplimiento de los compromisos no se estableció de forma sistemática hasta los años ochenta y no siempre se llevó a cabo.

Las condiciones físicas del local del teatro, muy deficientes, y el frío producían indisposiciones en los actores: Antonio Vico y Carolina

${ }^{5}$ Los compromisos de las compañías para la feria de San Juan sufrían la competencia con otras ciudades como Rioseco, Valladolid y Villalón. Por la festividad de San Pedro la marcha a Burgos era, y sigue siendo en la actualidad, bastante frecuente. 
Civili enfermaron en León. Los cronistas tomaron a broma la situación de Vico y aceptaron sin problemas la ausencia de Carolina Civili, que días más tarde sufrió una operación en Madrid.

No parece difícil defender la positiva influencia de las compañías de prestigio en la educación y los gustos del público. Si el número de actores, la abundancia de medios en el vestuario, los accesorios, el decorado, la iluminación, la música y el sonido, están condicionados por las posibilidades de los diferentes grupos e inciden en la calidad de la representación, la selección de los repertorios también lo hace. Algunas compañías se especializaban y las de Leonardo Pastor y Maximino Fernández, por ejemplo, programaban zarzuela grande frente a otras, como la Wenceslao Bueno, que solía representar teatro de verso.

Las relaciones de los grupos teatrales con la prensa fueron exquisitas: para los revisteros las compañías venían precedidas de buenos antecentes, quizás inventados; las representaciones eran de gran calidad; los actores, de los que sólo los protagonistas solían recibir comentarios, estaban acertados; y si había algún fallo en la escenografía o el vestuario era imputable al Ayuntamiento y su deficiente gestión. En contrapartida, las compañías pagaban sus anuncios en los periódicos; pero no siempre, ni todas. La razón de tanta bondad parece cifrarse en la idea generalizada de que el teatro era una forma de educar al pueblo ignorante, que en León no podía permitirse el lujo de asistir a las funciones. Muy graves debían ser los agravios cuando se incluía algún comentario negativo, como ocurrió con la bufa de Orejón, cuyas chocarrerías y ademanes groseros merecieron la indignación del público y el crítico.

No hay, en los documentos consultados, alusiones a la relación de las compañías con los asistentes, salvo las infrecuentes llamadas a escena en los éxitos y los regalos a los actores ${ }^{6}$. Las compensaciones de los grupos estribaban en la escenificación de alguna obra de autor leonés, la recitación o el canto de composiciones locales o la improvisación de textos alusivos dirigidos al público, en especial a las señoras. Por otra parte, numerosas notas de prensa avisaban a los jóvenes inexpertos de los muchos peligros que encubrían los encantos de las actrices y recordaban a los agentes del orden su deber de reprimir los insultos a los actores y actrices, por parte de ciertos desaprensivos. Apenas se dieron, o no se documentan, actitudes groseras del público.

${ }^{6}$ Es un lugar común la frialdad del público de León. 
Es difícil escoger lo mejor de las ciento treinta y cinco compañías que se documentan en los escenarios leoneses en la segunda mitad de la pasada centuria. Algunas tan prestigiosas como las de Maximino Fernández, Domingo Goyenechea o Vicente Yáñez no se incluyen en esta selección por ser escasísima la documentación y muy cortas las estancias. El orden de inclusión en este estudio es cronológico, porque no se destacan otros criterios diferenciadores.

En el cuadro que aparece a continuación se recogen las compañías seleccionadas, con datos sobre el número de funciones, fechas de representación y la composición del elenco:

\begin{tabular}{|c|c|c|c|c|}
\hline & COMPAÑÍAS & FUNCIONES & FECHAS & ACTORES \\
\hline 1 & Victorino Tamayo y Baus & 9 & San Juan, 1860 & 15 \\
\hline 2 & Enrique Sureda y Lesaca & 13 & San Juan, 1875 & 34 \\
\hline \multirow[t]{4}{*}{3} & Manuel Catalina y Rodríguez & 7 & enero 1879 & 19 \\
\hline & & 8 & marzo 1879 & \\
\hline & & 1 & enero 1884 & \\
\hline & & 4 & mayo 1884 & \\
\hline 4 & Rafael Calvo & 12 & San Juan, 1879 & 14 \\
\hline 5 & Antonio Vico y López & 12 & San Juan, 1882 & 13 \\
\hline 6 & Carolina Civili & 8 & Cuaresma, 1884 & 9 \\
\hline \multirow[t]{2}{*}{7} & Pablo López & 7 & julio 1891 & 7 \\
\hline & & 3 & febrero 1893 & 3 \\
\hline \multirow[t]{4}{*}{8} & Ricardo Ruiz & no consta & Carnaval, 1892 & 50 \\
\hline & & 1 & octubre 1892 & \\
\hline & & 11 & San Juan, 1895 & \\
\hline & & 3 & Julio 1896 & \\
\hline \multirow[t]{3}{*}{9} & Manuel Barrilaro del Valle & 14 & abril 1899 & 40 \\
\hline & & 11 & noviembre 1899 & 7 \\
\hline & & 21 & septiembre 1900 & 10 \\
\hline 10 & Enrique Sánchez de León & 5 & julio 1900 & no consta \\
\hline
\end{tabular}

El análisis de las compañías de más prestigio que actuaron en León en la segunda mitad de la pasada centuria llevaría a graves errores si se pretendieran extrapolar las conclusiones al resto. Las diferencias más importantes se refieren a:

- El aumento del precio de las entradas, que era un estímulo para un público de élite, que llenaba el teatro en aquellas ocasiones. 
- Las fechas de actuación: las de Enrique Sureda y Lesaca, VictorinoTamayo y Baus, Rafael Calvo, Antonio Vico y López y Ricardo Ruiz vinieron para la feria de San Juan, la más importante de la ciudad. La de la Civili llegó en Cuaresma, momento difícil entonces para el negocio teatral.

- La crítica, condicionada siempre por los antecedentes de la capital, era amable; pero llegaba a la exquisitez si había méritos suficientes. Augusto Villabrille, conocido como Clotaldo, y José Estrañi se permitían lujos de entendidos y no tenían reparos en sus comentarios, aunque se tratase de actores o compañías de renombre.

- El distinto trato del Ayuntamiento: la de Antonio Vico no pagó el precio de los alquileres ${ }^{7}$ y recibió una subvención. El propio municipio tomó la iniciativa para contactar con la de Ricardo Ruiz, hecho muy infrecuente.

- La intervención de jóvenes leoneses para la tramitación de algunos contratos, en el caso de Manuel Catalina y Antonio Vico.

- La relación con el público: no sólo se asistía con asiduidad a las buenas representaciones, sobre todo si eran zarzuelas, sino que se premiaban las actuaciones más acertadas con regalos, se reclamaba la presencia en escena, etc. Premios y llamadas eran lo único extraordinario, porque el público de León era amable y tolerante.

- Cuatro de las diez compañías escogidas repitieron las estancias: las de Catalina, Pablo López, Manuel Barrilaro y Ruiz ${ }^{8}$.

Puede hablarse en León de la presencia de agrupaciones de gran renombre. Y si el hecho no encuentra justificación en la categoría del teatro, ni en la abundancia del público, ni en su preparación, en general se les apreciaba en lo que valían. Es excepcional el caso de Rafael Calvo que marchó poco contento de la atención dispensada por el público.

${ }^{7}$ En León solía ser de cien reales diarios y seis para el conserje.

8 Algunas más lo hicieron; pero lo habitual era eludir los compromisos, acortar las estancias o no volver: «Y la que llega una vez, pocas más vuelve» ( $L a$ Lira, n. ${ }^{\circ} 44,16$ 06-1884: 4). 


\section{COMPAÑÍAS ${ }^{9}$}

\subsection{Compañía de Tamayo y Baus}

Tamayo y Baus era el responsable de la compañía, junto a Amalia Gutiérrez y Enrique Arjona.

A pesar de la gran importancia de este grupo no aparecen referencias en las actas municipales; pero el periódico El Esla ofreció detallada información, siendo la primera compañía de la que se tienen, en las fuentes consultadas, datos de prensa.

Anunciados desde finales de mayo, llegaron a la ciudad el catorce de junio de 1860, en vísperas de las fiestas de San Juan, para la temporada de ferias. La última reseña es de primeros de julio; hicieron nueve funciones.

En principio, no respondió como podía esperarse de una compañía de tan altos vuelos. El gran crítico Estrañi, que hacía las reseñas de El Esla, más que aludir a las representaciones, atacaba al público. El gran aficionado que era se sublevaba ante la ignorancia de unos espectadores que preferían la zarzuela a los dramas clásicos que se ofrecían: las últimas funciones fueron apoteósicas y Amalia Gutiérrez, en especial, recibió aplausos, llamadas a escena y regalos.

Estrañi no dudó en poner en entredicho la unifomidad interpretativa de Tamayo ni en aconsejar a la actriz Gutiérrez «estudio y aplicación» (El Esla, n. ${ }^{\circ}$ 54, 05-07-1860: 4).

\subsubsection{Componentes}

La prensa de León proporcionaba en ocasiones los datos de actores y actrices al principio de temporada. Se recogían, en primer lugar, los nombres del director, primera actriz y primer actor, primer actor có-

${ }^{9}$ Las fuentes documentales más importantes en este apartado son las actas municipales y la prensa de León, si bien hemos utilizado otros recursos para establecer las relaciones con las representaciones en otros lugares. 
mico, primer actor de carácter, damas jóvenes, primer galán joven, actriz cómica, etc. El número de actores de las compañías que actuaban en la corte, aunque no todo lo numerosas que sería de desear, se reducía en provincias, donde la escasez imponía la interpretación de distintos papeles por un solo actor o actriz.

Cinco actrices y diez actores formaron el elenco. Se destacaron siempre las actuaciones de Amalia Gutiérrez, Enrique Arjona, Victoriano Tamayo y Baus, a quien la crítica consideraba mejor actor cómico que dramático, y Córcoles. Pero también se alude a otros actores y actrices, como Aladro, Concha Marín y Lansac, entre las señoras, y Alisendo y Benedetti entre los actores.

La actriz Amalia Gutiérrez recibía los encendidos aplausos del público en la mayoría de las funciones. Su beneficio, en la despedida de la compañía, supuso el total éxito de la beneficiada por su papel de niña inocentona en Las citas a media noche.

En la comedia ¡Don Tomás!, además de la Gutiérrez, se destacó el actor Córcoles y hubo una ligera crítica negativa para García ${ }^{10}$, en su papel protagonista.

\subsubsection{Repertorio}

Se representaron: Los amantes de Teruel, Adriana, La bola de nieve, La campana de la Almudaina, El castillo de San Alberto, Las citas a media noche, ¡Don Tomás!, Dos amos para un criado, El hombre de mundo, Un huésped del otro mundo, Mal de ojo y Sullivan.

\subsection{Compañía de zarzuela de Enrique Sureda y Lesaca}

La denominación de las compañías estuvo muy condicionada por la preferencia que se daba a las zarzuelas. Algunas se anunciaban como de zarzuela y verso y limitaban las primeras a obritas en un acto o ni siquiera eso. Ésta sí era de zarzuela.

10 No hay otros datos de este actor. 
Llegó a León para la feria de San Juan y empezaron las representaciones el diecisiete de junio de 1875 y dieron la última el cuatro de julio del mismo año. Los abonos se contrataron para diez días y se dieron trece funciones, ampliación que supone que las actuaciones fueron exitosas; de hecho, de algunas funciones se dijo que se habían celebrado con un lleno completo.

Las relaciones con el Ayuntamiento no fueron buenas y hasta se llegó al cobro diario, pagado en el acto al conserje. Cuando ya la compañía había abandonado la ciudad se hizo constar en un acta, que en el beneficio a favor del asilo de la ciudad, el sobreprecio de las entradas no había repercutido en la cuenta final. Se señaló, sin embargo, la generosidad de la actriz Enriqueta Toda, que entregó el importe total del suyo para dicho asilo.

Las negociaciones con los ediles debieron retener todo el verano en la ciudad al empresario Sureda ${ }^{11}$. Los seculares incumplimientos de las compañías pretendieron evitarse con un documento que contemplaba la prevención de los desfalcos, la verificación de las recaudaciones y la distribución de las localidades. En agosto se firmó un contrato por dos años, pero nunca se llevó a cabo.

\subsubsection{Componentes}

Enrique Sureda y Lesaca era uno de los empresarios; dirigía la compañía José Sala Julién y el representante era José María Estrada.

La prensa hablaba del «numeroso personal» (Porvenir de León, $\mathrm{n} .^{\circ}$ 1219, 07-07-1875: 3): contaron con ocho actrices, diez actores y dieciséis coristas. Hay que destacar a Enriqueta Toda, considerada por muchos como una de las más ilustres actrices del siglo.

En Los comediantes de antaño los actores tuvieron mucho éxito y fueron llamados a escena. Fue muy popular en la ciudad el vallisoletano Ramón de la Guerra, cuyos rimados piropos a las leonesas eran muy celebrados por la prensa.

11 Se documenta su estancia en la inauguración de los locales del Recreo Artístico, el 22-08-1875. 


\subsubsection{Repertorio}

El barberillo de Lavapiés, Los comediantes de antaño, La conquista de Madrid, El diablo en el poder, Los diamantes de la corona, El dominó azul, Estebanillo, Jugar con fuego, Los madgyares, Marina, El postillón de la Rioja y La soirée de Cachupín.

\subsection{Compañía de Manuel Catalina y Rodríguez}

Esta compañía visitó León en dos ocasiones, en enero y marzo de 1879 , en su ida y vuelta a Asturias y en otras dos en enero y mayo de $1884^{12}$. Hubo siete funciones en la primera estancia, ocho en la segunda, una en la tercera y tres en la cuarta, estando la última a cargo de miembros desgajados del grupo.

El director, además de sus alabadas actuaciones, agradaba al público de León con la improvisación de ciertas quintillas y la subida a escena de obras de un autor local como La cartera de un pintor, de Hipólito Carreño.

El enjuiciamiento del grupo sobrepasaba las posibilidades críticas de nuestros periodistas, que se confesaban abrumados por tal responsabilidad. No obstante, el gran cronista Clotaldo no dudó en poner las cosas en su sitio: aunque en las primeras estancias se alabaron las representaciones, durante las de 1884 el público no fue tan fervoroso y se pretendió justificar las ausencias por razones climatológicas. Pero el comentarista no sólo afeó la conducta del grupo por abandonar en León parte de su elenco, que precisó de la caridad pública, sino que señaló la escasez de actores y calificó las actuaciones como regulares.

\subsubsection{Componentes}

Diecinueve miembros en total componían el elenco: ocho actrices, seis actores, dos apuntadores y el representante. De los que actuaban

12 Las razones de la salida a provincias de estos afamados grupos debía cifrarse en asuntos económicos, según la tesis de Espín Templado (1988). Las funciones por horas restaron la asistencia a otros espectáculos. 
unos años antes en Madrid, sólo el director y Matilde Fernández vinieron a León.

Se destacaron las actuaciones de las actrices Solís y Estrada y los señores Pastrana, Barta y Carrera.

\subsubsection{Repertorio}

El anzuelo, La campanilla de los apuros, Cómo se empieza, Consuelo, Echar la llave, Enmendar la plana a Dios, El gran filón, La ley del mundo, El nudo gordiano, La primera y la última, Sheridan, Sin cocinera y Vivir al día fueron las obras representadas en su primera actuación.

A primera sangre, La careta verde, La cartera de un pintor, La casa de fieras, Las citas a media noche, Consuelo, Las diabluras de Perico, La feria de las mujeres, El hombre de mundo, La levita, Un marido como hay muchos, No la hagas y no la temas, Novio a pedir de boca, El poeta de guardilla, El pañuelo blanco, Lo que sobra a mi mujer y El vecino de enfrente, se vieron en marzo y abril de 1879.

De la tercera estancia sólo se recogió una función en la que se puso en escena La Pasionaria.

Lluvia de oro, Marineros en tierra, La mariposa, No hay humo sin fuego, El octavo no mentir, Pepita y El rizo de doña Marta, se vieron en la cuarta.

\subsection{Compañía de Rafael Calvo}

Llegó a León el dieciséis de junio de 1879 y se despidió a finales del mismo mes. Acortó la estancia prevista por la escasa respuesta del público, marchando hacia Vitoria. Las fechas de las funciones son muy imprecisas y se proporcionan datos de doce.

\subsubsection{Componentes}

La compañía venía formada por cinco actrices y nueve actores; pero traía hasta un peluquero, lo que no era habitual en estos grupos. Cuatro 
de los miembros eran de apellido Calvo y dos de las actrices Calderón: es probable que se tratase de grupos familiares, frecuentes entre las gentes de teatro, aunque en la mayoría de los casos no hay datos, en lo referido a León, que avalen esta información.

\subsubsection{Repertorio}

Un drama nuevo, En el seno de la muerte y Tehudis, esta última de Sánchez de Castro, muy vinculado a León. No fue llamado el autor a escena, aunque estaba en la sala; el cronista, oponente suyo en cuestiones ideológicas, ironizó con enorme dureza en sus comentarios.

\subsection{Compañía de Antonio Vico y López}

Llegó para las fiestas de San Juan de 1882, tras las gestiones de jóvenes leoneses. Dada su categoría artística, recibió una subvención de dos mil reales y la exención del pago de los alquileres. El abono, que abarcaba del quince al treinta de junio, copó muchas entradas y los cronistas animaban al público para que ocupase las restantes. Pero la asistencia no se correspondió con las espectativas y Vico enfermaba de vez en cuando, aunque seguía anunciándose. Su comportamiento, documentado en otros escenarios, era la normal «actitud de dominio arbitrario del primer actor» (Espín Templado, 1988, I: 325) y acabó con la paciencia del público y de los críticos. Además no todas sus actuaciones gustaban: en La jura de Santa Gadea estuvo poco convincente en su papel del Cid.

Hubo once funciones y una más que se suspendió, después del primer acto, por indisposición del actor. Quizás tuviera un punto de razón, porque la tradición de la inasistencia de público llega a las crónicas actuales:

Las mejores anécdotas del teatro se han referido siempre a funciones con falta de público. Así, en cierta ocasión, el actor Antonio Vico estaba en escena, en una de sus visitas a León, y era tan escaso el público que llenaba, o, mejor dicho, no llenaba la sala, que en un momento en que otro 
actor debía simular que le hablaba en voz baja, Vico exclamó: ¡Puedes hablar alto; estamos solos! (Muñoz Sánchez, 1990: 10-11).

La compañía no cumplió en absoluto las expectativas del público y cometió además un error de cronología al iluminar el altar de La jura de Santa Gadea con velas de esperma. El gran periodista Clotaldo lo glosó con enorme ironía. Tampoco dudó el crítico en censurar su irregular conducta.

\subsubsection{Componentes}

Concepción Marín, Concepción Constan y Dolores Díaz, se destacaron entre las cinco actrices de la compañía. De los ocho actores, Vico era el divo indiscutible. No todas las funciones fueron del agrado del público y el actor Valero recibió varias amonestaciones; tampoco gustó Sánchez de León en El gran Galeoto.

\subsubsection{Repertorio}

El alcalde toreador, Amar sin dejarse amar, Cambio de vía, La capa de José, La casa de fieras, Con la música a otra parte, Consuelo, Echar la llave, El forastero, El gran Galeoto, Guzmán el Bueno, Un joven audaz, La jura de Santa Gadea, La levita, La ley suprema, Levantar muertos, La muerte civil, La muerte en los labios, El octavo no mentir, Suma y sigue, Un tigre de Bengala y La vida es sueño.

\subsection{Compañía de Carolina Civili}

Representó nueve funciones. El hecho de que fuese temporada de Cuaresma, con unos espectadores remisos en otros escenarios, justifica las dos peticiones del Teatro Municipal de León: uno de los solicitantes era Wenceslao Bueno; la otra, la prestigiosa Civili. La compañía no siempre contó con mucho público: 
La compañía en que figura la eminente trágica Sra. Civili, se ha ausentado de nuestra población, admirada del apartamiento que mostramos hacia los goces mundanos, llegada la época de Cuaresma (La Lira, n. ${ }^{\circ}$ 33, 31-03-1884: 2).

\subsubsection{Componentes}

Sólo vinieron cuatro actrices y cinco actores, además de una actriz infantil, Rosario Senra, que, como todos los niños, fue muy aplaudida ${ }^{13}$. Se alabaron también las actuaciones de Martínez, Romero y Val, en algunas funciones. La escenificación de La Pasionaria supuso duras críticas, con excepción de la actriz infantil. La Civili estuvo condicionada por su mala forma física en su actuación en Norma; pero el público supo entenderlo.

Varios miembros de su equipo compartieron la escena, en la última actuación, con los miembros de la Compañía Imperial Japonesa, de ejercicios de destreza y equilibrio.

\subsubsection{Repertorio}

Amor de madre, Dos hijos, En perpetua agonía, Hay entresuelo, Levantar muertos, La locura de amor, Mal de ojo, Norma, Noticia fresca, La Pasionaria, Robo en despoblado y El Trovador.

\subsection{Compañía de Pablo López}

Esta compañía llegó a León, por vez primera, en julio de 1891, dirigida por el tenor cómico Pablo López, de paso a Galicia. Dieron siete funciones.

13 Las compañías infantiles y la presencia de niños en los grupos de actores fueron legales hasta finales del siglo XIX y muy populares. 
Tres representaciones se recogieron en la segunda temporada, en febrero de 1893. Este grupo teatral viajaba por toda la península y se documentan funciones suyas hasta en Las Canarias.

\subsubsection{Componentes}

La prensa recogió la lista de actores que se componía de doce actrices, catorce actores y veinticuatro coristas, mucho más numerosa de lo que era habitual. Se destacaron las actuaciones del tenor Juan Beltrami, del barítono José Lacarra y de la primera tiple dramática Josefina Soriano.

En su segunda estancia, en febrero de 1893, se redujeron a tres actrices y ocho actores.

\subsubsection{Repertorio}

Entre el amplio repertorio que anunciaron figuraban varias óperas. En León se vieron las zarzuelas: La bruja, Catalina, Marina, El milagro de la Virgen, Pepe-Hillo, La tempestad y Un tesoro escondido.

En la segunda estancia interpretaron: Los madgyares y El molinero de Subiza.

\subsection{Compañía de zarzuela de Ricardo Ruiz}

Se le había cedido gratis el Teatro Municipal en el Carnaval de 1892 , en atención a la importancia de la compañía ${ }^{14}$. No sería muy adecuado su comportamiento cuando en su petición para la feria de San Juan de 1894 se le exigieron a la compañía tres condiciones: pago por adelantado, escenificación obligada de las diez representaciones

14 Todos los datos de esta primera estancia se reducen a un acta municipal donde consta la concesión. 
ofrecidas y que acudiese el elenco prometido. No vino entonces; y de las funciones de octubre sólo hay constancia de una. En las ferias de San Juan de 1895 se registraron once funciones y en julio de 1896, tres ${ }^{15}$. En medio de estas últimas actuaciones y respetando la concesión, se autorizaron unas funciones a la compañía infantil de Luis Abella.

\subsubsection{Componentes}

No hay datos de la primera venida; de la segunda sólo se citaba a Francisca Riotort, al bajo González, al barítono, a Mendizábal, a los actores Suárez y Garro y al director Reparaz. En la tercera, hay una lista completa de los componentes de la compañía que constaba de seis actrices, catorce actores y treinta coristas.

En 1895 dirigían la compañía Cosme Bauzá y Francisco Villegas.

\subsubsection{Repertorio}

En su venida de mayo de 1895 , publicaron un amplísimo repertorio que incluía también algunas óperas. En 1894 representaron en León: Las campanas de Carrión, La verbena de la Paloma o El boticario y las chulapas y celos mal reprimidos.

Los africanistas, La bruja, La Dolores, El dúo de la Africana, Marina, Miss Helyett Petitte, Música clásica, El rey que rabió, Sueños de oro, La tela de araña y La tempestad, se vieron en las funciones de junio de 1895. Repitieron La verbena de la Paloma o El boticario y las chulapas y celos mal reprimidos.

\subsection{Compañía de zarzuela de Manuel Barrilaro del Valle}

Además de la categoría artística del grupo, se agradecieron, en las notas de prensa, las funciones de caridad que se dieron: una de ellas, a favor de un pueblo del Bierzo destruido por el fuego, aquellos días;

15 Sólo se documentan datos de la concesión. 
otra, socorrió a varios artistas de una agrupación dramática que se encontraban en la ciudad sin medios para marchar. Iniciaron sus actuaciones en abril de 1899.

Estando la compañía en Galicia, en junio de 1900, el Ayuntamiento de León les ofreció el Teatro para la feria de San Juan; no hay constancia de representaciones en aquellos días. En septiembre se recibió una comunicación de la empresa, con unas peticiones inaceptables: sus argumentos aludían a la protección que recibían los artistas extranjeros y sus ganancias. La prensa resumía la respuesta de los ediles con un «buenas viñas hay en Toro» (Porvenir de León, n. ${ }^{\circ} 3819,12-09-1900: 3$ ).

A finales de 1900 pretendieron alquilar el Teatro de León por tres años: se comprometían a dar hasta noventa funciones anuales y al menos quince durante las ferias; el Ayuntamiento podría disponer de su palco y del edificio cuando fuese necesario; el personal, con excepción del conserje, correría a cargo de la compañía. Se comprometían además a entregar doscientas butacas, estucar el vestíbulo y enlosarlo de nuevo y decorar el salón alto. De no aceptarse la propuesta, solicitaban treinta funciones más. Se autorizaron las funciones y se aceptó el arriendo bajo quince nuevas condiciones. El uno de enero de 1901, incumplidos los acuerdos por la compañía, el municipio rescindió el contrato y pidió las llaves del edificio ${ }^{16}$.

La primera función de esta última estancia fue el veintitrés de septiembre de 1900; a partir del día veintisiete se anunciaron por horas y tuvieron muchísimo éxito. Se documentan veintiuna representaciones, que se extendieron hasta finales de octubre.

\subsubsection{Componentes}

En la primera estancia de abril de 1899 , diez actrices, diez actores y veinte coristas componían el grupo, del que se destacó a la actriz Pérez, en el papel masculino de Carlos, en La viejecita ${ }^{17}$. También se alabaron

16 Un documento del alcalde de Zamora responde al de León que se interesa por el paradero de Barrilaro. Informa de los incumplimientos en aquella ciudad y en Salamanca. Pero añade que « (...) el Sr. Barrilaro es, dentro del círculo en que viven los que a tal industria se dedican, el menos informal» (Archivo Histórico Municipal de León, Documento Teatro Principal, 14-06-1901).

17 Muy singular en la situación dada era la secular escasez de mujeres, tanto en compañías profesionales, como en las de aficionados. 
las actuaciones de las actrices Aurora Solís, Magariño, Marcos y Río; y de los actores Moya, Zambrano, Carrasco y Chanot. Cuando volvieron en noviembre se habían reducido a siete: tres actrices y cuatro actores; pero contaban con la presencia del «célebre transformista Giuseppe Minuto discípulo de Frégoli» (El Porvenir de León, n. ${ }^{\circ} 3734$, 18-11-1899: 3). También se resaltó la presencia de un nuevo tenor, Teodoro Ristorini.

\subsubsection{Repertorio}

Agua, azucarillos y aguardiente, La banda de trompetas, La buena sombra, El cabo primero, Los camarones, Campanero y sacristán, Caramelo, Certamen nacional, Los cocineros, Cuadros disolventes, La fiesta de San Antón, El grumete, Lucifer, El mantón de Manila, La marcha de Cádiz, La mujer del molinero, Las mujeres, El mundo comedia es o El baile de Luis Alonso, El organista, Pepe Gallardo, El primer reserva, Los puritanos, El rey que rabió, El santo de la Isidra, La viejecita y Las zapatillas, fueron los títulos de su primera venida.

En noviembre representaron: El cabo primero, Camaleonte, $\mathrm{Ca}$ ramelo, Campanero y sacristán, Cuadros disolventes, La czarina, El dúo de la Africana, En las astas del toro, La fiesta de San Antón, La Gran Via, Gota serrana, El grumete, La indiana, La maja, La medalla, Marina, Pepe Gallardo, Los presupuestos de Villapierde, Los puritanos, Relámpago, El señor Joaquín y La viejecita.

En septiembre de 1900 escenificaron: Agua, azucarillos y aguardiente, La alegría de la huerta, El barquillero, Los borrachos, La buena sombra, Los buenos mozos, El cabo primero, La cara de Dios, Caramelo, Carmela, Los cocineros, La czarina, el dúo de la Africana, Eclipse total, La feria de Sevilla, La fiesta de San Antón, Gigantes y cabezudos, El grumete, Instantáneas, La leyenda del monje, Joshé Martín el tamborilero, María de los Ángeles, Niña Pancha, El organista, Pepe Gallardo, El querer de la Pepa, El señor Joaquín, El traje de luces y El último chulo.

\subsection{Compañía de Enrique Sánchez de León}

Las reseñas de prensa recogieron cinco representaciones; pero la que se celebró con motivo del aniversario de la toma de Tarifa no 
incluía las obras subidas a escena y se limitaba a lamentar que no se hubiese representado el drama alusivo de Gil y Zárate.

En la función del día veinticinco de julio de 1900, después de La tía de Carlos y a falta de fin de fiesta, las actrices de la compañía bailaron unas sevillanas.

\subsubsection{Componentes}

Sólo se destacaron las actuaciones de Carlota Lamadrid, considerada como una de las más eminentes actrices de la pasada centuria.

\subsubsection{Repertorio}

Mancha que limpia, Pasionaria, Las personas decentes, Pobre porfiado, Remedios y La tía de Carlos.

\section{Referencias bibliográficas}

Alonso CORTÉs, Narciso (1947). El teatro en Valladolid en el siglo XIX. Valladolid: Imprenta Castellana.

Archivo Histórico Municipal de León, Documento Teatro Principal.

El Esla (1860).

Espín Templado, María del Pilar (1988). El teatro por horas en Madrid (1870-1910). Madrid: Universidad Complutense (T.D.).

FERNÁNDEZ GARCIA, Estefanía (1997). León y su actividad escénica en la segunda mitad del siglo XIX (Tesis doctoral). Madrid: UNED.

- (2000). El teatro en León en la segunda mitad del siglo XIX. León: Universidad de León (prólogo de José Romera Castillo).

FUENTE BALLESTEROS, Ricardo de la (1988). Introducción al teatro español del siglo XX (1900-1936). Valladolid: Aceña.

La Estafeta de León (1891).

La Legalidad (1882).

La Lira (1884). 


\section{Estefanía FernáNdez García}

La Montaña (1994-1995).

MuÑIZ SÁNCHEZ, José María (1990). «El lenguaje del teatro». El Diario de León, n. ${ }^{\circ}$ 39.958; Filandón, 10-06, 11 y 12.

Porvenir de León $(1875,1879,1899)$. 